\title{
The dynamics of an HIV/AIDS model with screened disease carriers
}

\author{
S.D. Hove-Musekwa ${ }^{\mathrm{a} *}$ and F. Nyabadza ${ }^{\mathrm{b}}$ \\ ${ }^{a}$ Department of Applied Mathematics, National University of Science and Technology, Bulawayo, \\ Zimbabwe; ${ }^{b}$ South African Center of Epidemiological Modelling and Analysis, University of Stellenbosch, \\ Stellenbosch, South Africa
}

(Received 18 February 2008; final version received 13 November 2008)

\begin{abstract}
The presence of carriers usually complicates the dynamics and prevention of a disease. They are not recognized as disease cases themselves unless they are screened and they usually spread the infection without them being aware. We argue that this has been one of the major causes of the spread of human immunodeficiency virus (HIV). We propose, in this paper, a model for the heterogeneous transmission of HIV/acquired immunodeficiency syndrome in the presence of disease carriers. The model allows us to assess the role of screening, as an intervention program that can slow the epidemic. A threshold value $\psi^{*}$, for the screening rate is obtained. It is shown numerically that if $80 \%$ or more of the carrier population is screened, the epidemic can be contained. The qualitative analysis is done in terms of the model reproduction number $R$. The model has two equilibria, the disease free equilibrium and a unique endemic equilibrium. The disease free equilibrium is globally stable of $R<1$ and the endemic equilibrium is is locally stable for $R>1$. A detailed discussion of the model reproduction number is given and numerical simulations are done to show the role of some of the important model parameters.
\end{abstract}

Keywords: epidemic model; carriers; screening; stability; reproduction number

AMS Subject Classification: 92B05; 92D30; 93D20; 34D23

\section{Introduction}

The acquired immunodeficiency syndrome (AIDS) epidemic is a serious, growing public health problem worldwide. The cause is known and the principal routes of transmission understood but resources for treating human immunodeficiency virus (HIV) infected patients and for combating the spread of the virus are limited. Public health planning continues to be hampered by uncertainties about key epidemiological parameters such as the typical duration and intensity of infectiousness; the fraction of those infected who progress to develop AIDS and the progression time. Control of the epidemic therefore depends on promoting behavioural change among the subgroups of populations where infection is taking place and on optimum use of available therapy for those infected. Both approaches require a quantitative understanding of the dynamics of the epidemic in the population. The basis for such an understanding is two-fold. Firstly, we need to generate information on the evolving state of HIV infection and HIV risk behaviour in the population, by the elaboration of appropriate monitoring systems, secondly, the development of mathematical models and methods, which can fully exploit the information generated.

\footnotetext{
*Corresponding author. Email: sdmusekwa@nust.ac.zw
} 
This highlights the need to develop mathematical models that help in the understanding of HIV/AIDS disease and help policy makers to make rational policy decisions.

Many mathematical models have been developed to monitor the epidemic and explore the impact of intervention strategies that are being implemented $[2,3,6,12,14,15,17,18,22]$. The most recent work on modelling the role of screening unaware infectives is given in Ref. [26]. This paper extends these models by incorporating carriers, some of whom will be screened. In addition, treatment of screened cases and individuals with AIDS is also incoporated. According to Ref. [1], a major complication of many diseases is the existence of carriers, i.e. individuals who, although apparently healthy themselves, are already infected and are capable of transmitting the infection to others. In fact, they are not themselves usually recognized as actual cases. The potential danger posted by carriers is apparent since they spread infection without the awareness of their disease status and are usually not under any disease surveillance. However, the exact role carriers play in the transmission dynamics of the disease is not so easy to predict or pinpoint. It is of great epidemiological significance to investigate, qualitatively and quantitatively, the role of disease carriers in the transmission dynamics using mathematical models. The role of carriers may be significant in the AIDS epidemic, especially in the light of new HIV drug developments and various intervention programmes.

Upon infection by HIV, an individual enters the primary (acute) HIV infection stage. Some people experience very strong symptoms upon contraction of HIV, while others experience none at all. Because of the non-specificity of symptoms of primary HIV infection, diagnosis and identification of cases depends on individuals' understanding of HIV infection dynamics after risky sexual contacts. Primary HIV infection can, but does not always, progress to early symptomatic HIV infection that can eventually advance to AIDS [20,24]. We, however, assumed in this paper that primary HIV infection leads to symptomatic HIV infection. Once primary infection is over, individuals become symptomatic for 5-10 years. If no screening leading to treatment is done, individuals eventually progress to AIDS.

Highly active antiretroviral therapy (HAART) is a very potent combination of antiretroviral (ARV) drugs, that can significantly prolong HIV infected patients' lives. The negative effect of drug treatment may be that partially recovered patients can resort back to risky sexual behaviour and hence undermine the effectiveness of the drug over the whole population. Although under HAART the proportion of primary resistance cases decreases transiently, the epidemic worsens because the actual numbers of infected individuals and of drug resistant carriers increases [21]. This is because of the increase in risky behaviour, both on the part of the newly infected who has higher viral loads and is more likely to transmit the virus, and also the carriers who are unaware of their HIV status. Therefore qualitative studies, like this one, on how carriers can impact the AIDS transmission dynamics in the population are important for the evaluation of the impact of HIV treatment and education campaigns. The main focus is on determining the effects of carriers and randomly screened carriers, who are aware of their status, on the transmission of HIV. The results of this study have implications on the validity of the parameters estimated for models without carriers. We formulate the model as a system of ordinary differential equations in Section 2 and the analysis of the model is given in Section 3. By setting some parameters to zero, the model exhibits various scenarios, such as model without carriers and model with no intervention, that are presented and discussed in Section 4. Numerical simulations of the model are done in Section 5 and some concluding remarks are made in the last section.

\section{Model formulation}

A population of size $N(t)$, which varies with time, is divided into seven classes consisting of individuals who are susceptible to the disease $S(t)$, infectious and symptomatic primary HIV 
infectives $I(t)$, whom we will call normal infectives, carriers of the disease who are asymptomatic and infectious $C(t)$, randomly screened carriers $C_{\mathrm{s}}$, under HIV treatment $T$, with full blown AIDS $A(t)$ and those with AIDS but under treatment $A_{\mathrm{t}}$. The total population is thus given by $N(t)=S(t)+I(t)+C(t)+C_{\mathrm{s}}(t)+T(t)+A(t)+A_{\mathrm{t}}(t)$. The model incorporates the transmission of HIV infection by the normal infectives, carriers, treated individuals and those who have full blown AIDS, i.e. those in the classes $I(t), C(t), T(t)$ and $A(t)$, respectively. Primary HIV infection is assumed to be symptomatic following [19] and the references cited therein (see also, Primary HIV infection and post exposure prevention. Available at http://www.ccspublishing.com/journals2a/ Primary_HIV.html and HIV early symptoms. Available at http://www.hivsymtomsonline.com/ hiv-early-symptoms.html). However, the duration of infectivity is short [8]. Soon after primary infection, individuals can either become asymptomatic with respect to HIV infection or are given HAART, if detected early. The asymptomatic HIV infectives are regarded as carriers and those on HAART join the class of those under treatment. Those in the class of carriers are subjected to screening. However, we assume that those who have been screened from the carrier class will not transmit the disease since they are counselled during screening. The counselling is assumed to be very effective in preventing high-risk sexual behaviour. Those in the $A_{\mathrm{t}}$ class are also assumed not to engage in HIV spreading activities. The population mixes homogeneously. This means that susceptible individuals are equally likely to be infected by an infectious individual in the case of a contact.

We give a brief description of the model parameters used in the model. We firstly consider the probability of infection $\beta$. This is the probability of transmission from an infectious individual to a susceptible individual which is formed from two components, namely the likelihood of close contact between two individuals, such that transmission can occur (dependent upon the pattern of mixing in the population) and the probability that transmission will occur as a result of the close contact (dependent upon the innate contagiousness of the infectious organism and perhaps on the genetic or behavioural susceptibility of the individual host). The probability of infection is significant in measuring the force of infection, i.e. the per capita rate at which susceptible individuals acquire infection. $\beta$ is difficult to determine since it is related to attitude factors and therefore an individual's risk of acquiring HIV is to a large extent determined by an individual's attitude and the role played during sexual intercourse [2]. Suppose $p$ is the probability of transmission per contact, the probability that a susceptible individual will not be infected by a single contact with an infected individual is $1-p$. Therefore, the probability that infection is avoided, when $n$ contacts have been made is $(1-p)^{n}$, giving us the transmission probability per partner $\beta=1-(1-p)^{n}$ [18]. We assume that $\beta_{1}, \beta_{2}, \beta_{3}$ and $\beta_{4}$ are the probabilities of infection by the symptomatic infectives, unscreened carriers and individuals under HIV treatment, respectively. However, we assume that the probability of infection of a susceptible by an individual in the primary symptomatic stage, $\beta_{1}$, is greater than all the other infection probabilities [7]. The probabilities thus satisfy the following relation, $\beta_{1}>\beta_{4}>\beta_{2}>\beta_{3}$.

Also of importance is the average number of sexual partners $k$ an individual can have. The mean number of sexual partners is a key parameter for epidemiological projections. Sexual partnerships and disease spread are co-evolving dynamic processes and the number of sexual partners is important for the determination of epidemic thresholds. The rate of acquisition of new partners depends largely on social and environmental factors that determine the living conditions, resources and social opportunities [9]. Cultural and religious beliefs have an influence on the number of new partners one can acquire. In some cultural settings, men are allowed to have as many partners as they wish and this has a significant impact on the value of $k$. Many people indulge in risky behaviours due to poverty, need to get financial support, revenge for having been infected unjustifiably and lack of knowledge on disease dynamics [13]. The total 
number of new symptomatic infectious individuals, at time $t$ is given by

$$
k\left(\beta_{1} \frac{I(t)}{N(t)}+\beta_{2} \frac{C(t)}{N(t)}+\beta_{3} \frac{T(t)}{N(t)}+\beta_{4} \frac{A(t)}{N(t)}\right) S(t) .
$$

The introduction of triple-drug therapies has lead to a subsequent increase in risky sexual behaviour, which in turn has lead to an increase in the number of new HIV infections [16]. Risk behaviour change can be measured by measuring the trends of the value of $k$, with a decline in the value of $k$ indicating an increase in behavioural change.

In a bid to model specific forms of interventions, we include the intervention rates $\gamma$ and $\psi$. The rate at which carriers are screened $\psi$, will largely depend on individuals wanting to know their status. This is also based on the anticipated potential benefits of screening. It is thus important to promote the screening of carriers. $\psi$ is a measure of the success of voluntary testing campaigns that are being advocated in the fight against the HIV/AIDS pandemic, especially in sub-Saharan Africa. The rates at which the infected seek treatment are given by $\gamma_{i}$, where $i=1, \ldots, 3$, for those in the normal infective class, the screened carriers and the AIDS classes, respectively.

Assuming that all those recruited into the population are susceptible, we can assume a constant recruitment rate $\Pi$. This rate of recruitment also covers juveniles who become sexually active. We assume heterosexual transmission of the disease with no vertical transmission, blood transfusion and needle sharing. We also assume that there is no recruitment of individuals resistant to HIV. In the absence of a disease, individuals in a population die of natural causes, assumed to occur at a constant rate $\mu$. Diseases such as HIV/AIDS impact mortality rates and it is always plausible to assume that individuals with AIDS die from both natural causes and from the disease. We assume disease-caused mortality rates $\delta_{1}$ and $\delta_{2}$ for individuals with AIDS, and are under no treatment and those with AIDS but under treatment, respectively.

We assume individuals progress from the normal infectives to become carriers at a rate $\sigma$. Variable disease progression rates between individuals have been documented, with progression categorized as rapid, intermediate and long-term non-progression [10]. Individuals progress from the normal infectives, carriers, screened carriers and treated classes to the AIDS class at rates $\rho_{i}$, where $i=1, \ldots, 4$, respectively. Our model is governed by the following system of nonlinear ordinary differential equations

$$
\begin{array}{ll}
\frac{\mathrm{d} S}{\mathrm{~d} t}=\Pi-\lambda(I, C, T, A) S-\mu S, & S(0)=S_{0}, \\
\frac{\mathrm{d} I}{\mathrm{~d} t}=\lambda(I, C, T, A) S-\left(\mu+\sigma+\rho_{1}+\gamma_{1}\right) I, & I(0)=I_{0}, \\
\frac{\mathrm{d} C}{\mathrm{~d} t}=\sigma I-\left(\mu+\rho_{2}+\psi\right) C, & C(0)=C_{0}, \\
\frac{\mathrm{d} C_{\mathrm{s}}}{\mathrm{d} t}=\psi C-\left(\mu+\rho_{3}+\gamma_{2}\right) C_{\mathrm{s}}, & C_{\mathrm{s}}(0)=C_{\mathrm{s} 0}, \\
\frac{\mathrm{d} T}{\mathrm{~d} t}=\gamma_{1} I+\gamma_{2} C_{\mathrm{s}}-\left(\mu+\rho_{4}\right) T, & T(0)=T_{0}, \\
\frac{\mathrm{d} A}{\mathrm{~d} t}=\rho_{1} I+\rho_{2} C+\rho_{3} C_{\mathrm{s}}+\rho_{4} T-\left(\mu+\gamma_{3}+\delta_{1}\right) A, & A(0)=A_{0}, \\
\frac{\mathrm{d} A_{\mathrm{t}}}{\mathrm{d} t}=\gamma_{3} A-\left(\mu+\delta_{2}\right) A_{\mathrm{t}} ; & A_{\mathrm{t}}(0)=A_{\mathrm{t} 0},
\end{array}
$$

where

$$
\lambda(I, C, T, A)=k\left(\beta_{1} \frac{I}{N}+\beta_{2} \frac{C}{N}+\beta_{3} \frac{T}{N}+\beta_{4} \frac{A}{N}\right) .
$$


From the summation of equations in system (1), we have the rate at which the total population is changing,

$$
\frac{\mathrm{d} N}{\mathrm{~d} t}=\Pi-\mu N-\delta_{1} A-\delta_{2} A_{\mathrm{t}}, \quad N(0)=N_{0} .
$$

In the absence of AIDS related deaths or individuals with AIDS, the population size approaches $\Pi / \mu$ as $t \rightarrow \infty$. It can be shown that for the system (1), the region

$$
\Gamma=\left\{\left(S, I, C, C_{\mathrm{s}}, T, A, A_{\mathrm{t}}\right) \mid\left\{S, I, C, C_{\mathrm{s}}, T, A, A_{\mathrm{t}}\right\} \geq 0, N \leq \frac{\Pi}{\mu}\right\}
$$

is positively invariant, making sure that the model is well posed and biologically meaningful. All dependent variables and the parameters are taken to be non-negative. We can omit the last equation of (1), since the $A_{\mathrm{t}}$ compartment is not involved in the dynamics of the disease.

\section{Model analysis}

In this section, we look at the equilibria and the qualitative features of the model by carrying out the stability analysis of the model. This analysis will enable us to determine the threshold conditions for the persistence or eradication of the disease.

\subsection{Model steady states}

The model has steady (equilibrium) states obtained by setting the right hand sides of system (1) to zero such that

$$
\begin{aligned}
& \Pi-\lambda^{*} S^{*}-\mu S^{*}=0, \\
& \lambda S^{*}-\left(\mu+\sigma+\rho_{1}+\gamma_{1}\right) I^{*}=0, \\
& \sigma I^{*}-\left(\mu+\rho_{2}+\psi\right) C^{*}=0, \\
& \psi C^{*}-\left(\mu+\rho_{3}+\gamma_{2}\right) C_{\mathrm{s}}^{*}=0, \\
& \gamma_{1} I^{*}+\gamma_{2} C_{\mathrm{s}}^{*}-\left(\mu+\rho_{4}\right) T^{*}=0, \\
& \rho_{1} I^{*}+\rho_{2} C^{*}+\rho_{3} C_{\mathrm{s}}^{*}+\rho_{4} T^{*}-\left(\mu+\gamma_{3}+\delta_{1}\right) A^{*}=0, \\
& \gamma_{3} A^{*}-\left(\mu+\delta_{2}\right) A_{\mathrm{t}}^{*}=0,
\end{aligned}
$$

where $\lambda^{*}=\lambda\left(I^{*}, C^{*}, T^{*}, A^{*}\right)$. From (2), the steady state expressions for $C^{*}, C_{\mathrm{s}}^{*}, T^{*}$ and $A^{*}$ in terms of $I^{*}$ are given by,

$$
C^{*}=\omega_{1} I^{*}, \quad C_{\mathrm{s}}^{*}=\omega_{2} I^{*}, \quad T^{*}=\omega_{3} I^{*} \text { and } A^{*}=\omega_{4} I^{*}
$$

where,

$$
\begin{aligned}
& \omega_{1}=\frac{\sigma}{\mu+\rho_{2}+\psi}, \quad \omega_{2}=\frac{\psi}{\mu+\gamma_{2}+\rho_{3}} \omega_{1}, \\
& \omega_{3}=\frac{\gamma_{1}\left(\mu+\gamma_{2}+\rho_{3}\right)\left(\mu+\rho_{2}+\psi\right)+\gamma_{2} \sigma \psi}{\left(\mu+\rho_{4}\right)\left(\mu+\gamma_{2}+\rho_{3}\right)\left(\mu+\rho_{2}+\psi\right)} \text { and } \\
& \omega_{4}=\frac{1}{\mu+\gamma_{3}+\delta_{1}}\left(\rho_{1}+\omega_{1} \rho_{2}+\omega_{2} \rho_{3}+\omega_{3} \rho_{4}\right) .
\end{aligned}
$$


From (3), we also have

$$
\lambda^{*}=\phi \frac{I^{*}}{N^{*}}, \text { where } \phi=k\left(\beta_{1}+\beta_{2} \omega_{1}+\beta_{3} \omega_{3}+\beta_{4} \omega_{4}\right) .
$$

Substituting for $\lambda^{*}$ in the second equation of (2), we have either $I^{*}=0$ or

$$
\phi S^{*}-\left(\mu+\sigma+\rho_{1}+\gamma_{1}\right) N^{*}=0 .
$$

From (4), we have

$$
\frac{S^{*}}{N^{*}}=\frac{\mu+\sigma+\rho_{1}+\gamma_{1}}{\phi} .
$$

The total population $N$ at the steady state can be written as

$$
N^{*}=S^{*}+\xi I^{*}, \text { where } \xi=1+\omega_{1}+\omega_{2}+\omega_{3}+\omega_{4} .
$$

Substituting for $N^{*}$ in (4), we obtain

$$
I^{*}=\left(\frac{R-1}{\xi}\right) S^{*}, \text { where } R=\frac{\phi}{\mu+\sigma+\rho_{1}+\gamma_{1}} .
$$

Substituting (3) and (5) in the first equation of (2) gives

$$
S^{*}=\frac{\Pi R \xi}{R \mu \xi+\phi(R-1)} .
$$

The first case $I^{*}=0$, results in the disease-free equilibrium point. This scenario corresponds to the situation, where there are no infectious individuals in the population who interact with the susceptible. The system thus has a disease-free equilibrium point given by, $E_{0}=((\Pi / \mu), 0,0,0$, $0,0)$. In this case, the population size approaches the steady state value $\Pi / \mu$.

The second case gives the endemic equilibrium point, $E_{1}=\left(S^{*}, I^{*}, C^{*}, C_{\mathrm{s}}^{*}, T^{*}, A^{*}\right)$, where

$$
\begin{aligned}
S^{*} & =\frac{\Pi R \xi}{R \mu \xi+\phi(R-1)}, \quad C^{*}=\omega_{1} I^{*}, C_{\mathrm{s}}^{*}=\omega_{2} I^{*}, \\
I^{*} & =\left(\frac{R-1}{\xi}\right)\left(\frac{\Pi R \xi}{R \mu \xi+\phi(R-1)}\right), \quad T^{*}=\omega_{3} I^{*}, A^{*}=\omega_{4} I^{*} .
\end{aligned}
$$

It is important to note that we can determine the expression for $A_{\mathrm{t}}^{*}$ from $A_{\mathrm{t}}^{*}=\left(\gamma_{3} /\left(\mu+\delta_{2}\right)\right) A^{*}$. We thus have the following result on the existence of the endemic equilibrium point.

THEOREM 3.1. If $R \leq 1$, system (1) has a unique equilibrium point $E_{0}$ as the only equilibrium point. However, if $R>1$, there exists a unique endemic equilibrium point $E_{1}$ in the interior of $\Gamma$, whose coordinates are given by (6).

$R$ is thus a threshold quantity, the model reproduction number. This is defined as the mean number of secondary cases generated by a typical infective in a population that is entirely susceptible. The existence of an endemic equilibrium point is used to determine the model reproduction number $R$. We discuss the model reproduction number and the local stability of the disease free point $E_{0}$ in the next subsection. 


\subsection{The reproduction number and local stability of $\mathbf{E}_{0}$}

The reproduction number is a key parameter, which determines the behaviour of the model. The model reproduction number can also be determined by the decomposition technique in Ref. [29]. A reproduction number obtained in this way determines the local stability of the disease free equilibrium point with local asymptotic stability for $R<1$ and instability for $R>1$ [11]. The technique is based on the fact that the reproduction number cannot be determined from the structure of the model alone but also on the decomposition of the model into infected and uninfected compartments. The ability to distinguish new infections from other changes being tracked in the model becomes crucial. Using matrix theory, $R$ is the spectral radius of the next generation matrix for the model. This means that $R$ tracks the growth and decline of successive generations of infectives when the epidemic begins. If $R<1$, then the disease free equilibrium is locally asymptotically stable, leading to the eradication of the disease. However, if $R>1$, the disease free equilibrium is not stable signalling an epidemic outbreak [5]. For a detailed analysis on the decomposition technique, we refer the readers to Ref. [29].

This implies that the long-term expected average number of secondary cases per generation produced by an infected individual is,

$$
R=R_{0 I}+\nu_{1} R_{0 C}+\nu_{2} R_{0 T}+\nu_{3} R_{0 A}
$$

where,

$$
\begin{aligned}
& R_{0 I}=\frac{k \beta_{1}}{\mu+\sigma+\rho_{1}+\gamma_{1}}, \quad R_{0 C}=\frac{k \beta_{2}}{\mu+\rho_{2}+\psi}, \\
& R_{0 T}=\frac{k \beta_{3}}{\mu+\rho_{4}}, \quad R_{0 A}=\frac{k \beta_{4}}{\mu+\gamma_{3}+\delta_{1}}
\end{aligned}
$$

and

$$
\begin{aligned}
\nu_{1}= & \left(\frac{\sigma}{\mu+\sigma+\rho_{1}+\gamma_{1}}\right) \\
\nu_{2}= & \left(\frac{\gamma_{1}}{\mu+\sigma+\rho_{1}+\gamma_{1}}\right)+\nu_{1}\left(\frac{\gamma_{2}}{\mu+\gamma_{2}+\rho_{3}}\right)\left(\frac{\psi}{\mu+\rho_{2}+\psi}\right) \\
\nu_{3}= & \frac{\rho_{1}}{\mu+\sigma+\rho_{1}+\gamma_{1}}+\nu_{1}\left[\left(\frac{\rho_{2}}{\mu+\rho_{2}+\psi}\right)+\left(\frac{\rho_{3}}{\mu+\rho_{3}+\gamma_{2}}\right)\left(\frac{\psi}{\mu+\rho_{2}+\psi}\right)\right] \\
& +\nu_{2}\left(\frac{\rho_{4}}{\mu+\rho_{4}}\right) .
\end{aligned}
$$

$R_{0 I}$ is the reproduction number due to infectives $I, R_{0 C}$ is the reproduction number due to the carriers $C, R_{0 T}$ is the reproduction number due to the treated individuals $T$ and $R_{0 A}$ is the reproduction number due to the individuals with full blown AIDS $A$. We can thus state the following result.

THEOREM 3.2. The disease free equilibrium point $E_{0}$ is locally asymptotically stable, if $R<1$ and unstable for $R>1$.

The terms in (7) can be explained as follows:

- $1 /\left(\mu+\sigma+\rho_{1}+\gamma_{1}\right), 1 /\left(\mu+\rho_{2}+\psi\right), 1 /\left(\mu+\gamma_{2}+\rho_{3}\right), 1 /\left(\mu+\rho_{4}\right), 1 /\left(\mu+\gamma_{3}+\delta_{1}\right)$ are the average times an individual spends in, the infected class $I$, the carriers class $C$, the screened carriers class $C_{\mathrm{s}}$, the teaded class $T$ and the AIDS class $A$. 
- $\sigma /\left(\mu+\sigma+\rho_{1}+\gamma_{1}\right)$ is the proportion of individuals who become carriers by progression from compartment $I$ to compartment $C$.

- $\gamma_{1} /\left(\mu+\sigma+\rho_{1}+\gamma_{1}\right)$ is the proportion of individuals who seek treatment and progress from compartment $I$ to compartment $T$.

- $\rho_{1} /\left(\mu+\sigma+\rho_{1}+\gamma_{1}\right)$ is the proportion of individuals who develop AIDS from compartment $I$.

- $\rho_{2} /\left(\mu+\rho_{2}+\psi\right)$ is the proportion of carriers who develop AIDS.

- $\rho_{4} /\left(\mu+\rho_{4}\right)$ is the proportion of individuals under treatment who develop AIDS.

It is interesting to note that the following expressions can equally be interpreted in a similar way even though they are products. This will give a clearer interpretation of $\nu_{2}$ and $\nu_{4}$.

- $\left(\rho_{2} /\left(\mu+\psi+\rho_{2}\right)\right)\left(\sigma /\left(\mu+\sigma+\rho_{1}+\gamma_{1}\right)\right)$ is the proportion of those individuals who develop AIDS from compartment $C$ having come from compartment $I$.

- $\left(\rho_{4} /\left(\mu+\rho_{4}\right)\right)\left(\gamma_{1} /\left(\mu+\sigma+\rho_{1}+\gamma_{1}\right)\right)$ is the proportion of those individuals who develop AIDS from compartment $T$ having come from compartment $I$.

- $\left(\sigma /\left(\mu+\sigma+\rho_{1}+\gamma_{1}\right)\right)\left(\psi /\left(\mu+\rho_{2}+\psi\right)\right)\left(\rho_{3} /\left(\mu+\rho_{3}+\gamma_{2}\right)\right)$ is the proportion of individuals who develop AIDS having started in compartment I via compartments $C$ and $C_{\mathrm{s}}$.

- $\left(\sigma /\left(\mu+\sigma+\rho_{1}+\gamma_{1}\right)\right)\left(\psi /\left(\mu+\rho_{2}+\psi\right)\right)\left(\gamma_{2} /\left(\mu+\gamma_{2}+\rho_{3}\right)\right)\left(\rho_{3} /\left(\mu+\rho_{3}+\gamma_{2}\right)\right)$ is the proportion of individuals who develop AIDS having started in compartment $I$ via compartments $C, C_{\mathrm{s}}$ and $T$.

\subsection{Global stability of the disease-free equilibrium}

To prove globally stability of the disease-free equilibrium point, we use the approach in Ref. [25]. For a bounded real-valued function $g$ on $[0, \infty)$, we define

$$
g_{\infty}=\lim _{t \rightarrow \infty} \inf g(t), \quad g^{\infty}=\lim _{t \rightarrow \infty} \sup g(t),
$$

and therefore, we state the following lemma.

Lemma 3.3. Let $g:[0, \infty) \rightarrow \Re$ be bounded and twice differentiable with a bounded second derivative. Let $t_{n} \rightarrow \infty$ and $g\left(t_{n}\right)$ converges to $g_{\infty}$ or $g^{\infty}$, for $n \rightarrow \infty$ then, $g^{\prime}\left(t_{n}\right) \rightarrow 0, n \rightarrow \infty[25]$.

THEOREM 3.4. If $R<1$, then $E_{0}$ is globally asymptotically stable.

Proof. Suppose $R<1$ and we choose a sequence $t_{1 n} \rightarrow \infty$, such that $C\left(t_{1 n}\right) \rightarrow C^{\infty}$ and $\mathrm{d} C\left(t_{1 n}\right) / \mathrm{d} t \rightarrow 0$ as $n \rightarrow \infty$, then

$$
C^{\infty} \leq \omega_{1} I^{\infty}
$$

The sequence $\left\{t_{1 n}\right\}$ is chosen in such a way that $C\left(t_{1 n}\right)$ converges to $C^{\infty}$ as $n \rightarrow \infty$. Choosing a second sequence $t_{2 n} \rightarrow \infty$, such that $C_{\mathrm{s}}\left(t_{2 n}\right) \rightarrow C_{\mathrm{s}}^{\infty}$, we have

$$
C_{\mathrm{s}}^{\infty} \leq \omega_{2} I^{\infty}
$$

Choosing a third sequence $t_{3 n} \rightarrow \infty$, such that $A\left(t_{3 n}\right) \rightarrow A^{\infty}$, we have

$$
A^{\infty} \leq \omega_{4} I^{\infty} .
$$


A fourth sequence $t_{4 n} \rightarrow \infty$, such that $T\left(t_{4 n}\right) \rightarrow T^{\infty}$ will enable us to write the following relation

$$
T^{\infty} \leq \omega_{3} I^{\infty}
$$

Finally, choosing a fifth sequence $t_{5 n} \rightarrow \infty$, such that $I\left(t_{5 n}\right) \rightarrow I^{\infty}$ and $\mathrm{d} I\left(t_{5 n}\right) / \mathrm{d} t \rightarrow 0$. Substituting Equations (8)-(11) into the second equation of (1), we have

$$
\phi \frac{I^{\infty}}{N^{*}} S^{*}-\left(\mu+\sigma+\rho_{1}+\gamma_{1}\right) I^{\infty} \geq 0
$$

Thus,

$$
\left(\mu+\sigma+\rho_{1}+\gamma_{1}\right)(R-1) I^{\infty} \geq 0 .
$$

Since $R<1$, it implies that $I^{\infty} \leq 0$, which is only possible if $I^{\infty}=0$, but $I_{\infty} \geq 0$ and thus, we have $I^{\infty}=I_{\infty}=0 \Rightarrow I(t) \rightarrow 0$ as $t \rightarrow \infty$. From (8) to (11), we have $C(t), T(t)$ and $A(t) \rightarrow 0$ as $t \rightarrow \infty$.

We thus have, from the equation $\mathrm{d} N / \mathrm{d} t=\Pi-\mu N-\delta A, N_{\infty}=\Pi / \mu$. But for $N>\Pi / \mu$, $\mathrm{d} N / \mathrm{d} t \leq 0$. We therefore consider the solution for which $N^{\infty} \leq \Pi / \mu$, which gives $N_{\infty}=N^{\infty}=\Pi / \mu$. Hence, $E_{0}$ is globally asymptotically stable.

\subsection{Local stability of the endemic equilibrium}

The standard technique of determining the local stability of the endemic equilibrium is by finding the eigenvalues of the Jacobian matrix evaluated at the endemic equilibrium from which one can infer the stability of the equilibrium point. Such an approach would be mathematically cumbersome for system (1). We therefore resort to the centre manifold theory presented in Refs. [4,23,29]. In particular, we use theorem 4.1 in Ref. [4] which was reproduced in Ref. [23]. We rewrite system (1) by letting the variables $S=x_{1}, I=x_{2}, C=x_{3}, C_{\mathrm{s}}=x_{4}, T=x_{5}$ and $A=x_{6}$. We also let $\eta_{1}=\mu+\sigma+\rho_{1}+\gamma_{1}, \eta_{2}=\mu+\rho_{2}+\psi, \eta_{3}=\mu+\gamma_{2}+\rho_{3}, \eta_{4}=\mu+\rho_{4}$ and $\eta_{5}=\mu+\gamma_{3}+\delta_{1}$. In vector form, system (1) is of the form $\mathrm{d} \mathbf{X} / \mathrm{d} t=\mathcal{F}(\mathbf{X})$ where $\mathbf{X}=\left(x_{1}, x_{2}, x_{3}, x_{4}, x_{5}, x_{6}\right)^{\mathrm{T}}$ and $(\cdot)^{\mathrm{T}}$ denotes the matrix transpose. We can thus write the system as

$$
\begin{aligned}
\frac{\mathrm{d} x_{1}}{\mathrm{~d} t} & =\Pi-k\left(\frac{\beta_{1} x_{2}+\beta_{2} x_{3}+\beta_{3} x_{5}+\beta_{4} x_{6}}{\sum_{i=1}^{6} x_{i}}\right) x_{1}-\mu x_{1}:=f_{1}, \\
\frac{\mathrm{d} x_{2}}{\mathrm{~d} t} & =k\left(\frac{\beta_{1} x_{2}+\beta_{2} x_{3}+\beta_{3} x_{5}+\beta_{4} x_{6}}{\sum_{i=1}^{6} x_{i}}\right) x_{1}-\eta_{1} x_{2}:=f_{2}, \\
\frac{\mathrm{d} x_{3}}{\mathrm{~d} t} & =\sigma x_{2}-\eta_{2} x_{3}:=f_{3}, \\
\frac{\mathrm{d} x_{4}}{\mathrm{~d} t} & =\psi x_{3}-\eta_{3} x_{4}:=f_{4}, \\
\frac{\mathrm{d} x_{5}}{\mathrm{~d} t} & =\gamma_{1} x_{2}+\gamma_{2} x_{4}-\eta_{4} x_{5}:=f_{5}, \\
\frac{\mathrm{d} x_{6}}{\mathrm{~d} t} & =\rho_{1} x_{2}+\rho_{2} x_{3}+\rho_{3} x_{4}+\rho_{4} x_{5}-\eta_{5} x_{6}:=f_{6} .
\end{aligned}
$$


The Jacobian matrix of (13) at the disease free equilibrium point is given by

$$
J_{E_{0}}=\left(\begin{array}{cccccc}
-\mu & -k \beta_{1} & -k \beta_{2} & 0 & -k \beta_{3} & -k \beta_{4} \\
0 & k \beta_{1}-\eta_{1} & k \beta_{2} & 0 & k \beta_{3} & k \beta_{4} \\
0 & \sigma & -\eta_{2} & 0 & 0 & 0 \\
0 & 0 & \psi & -\eta_{3} & 0 & 0 \\
0 & \gamma_{1} & 0 & \gamma_{2} & -\eta_{4} & 0 \\
0 & \rho_{1} & \rho_{2} & \rho_{3} & \rho_{4} & -\eta_{5}
\end{array}\right) .
$$

We can also obtain the model reproduction number $R$ from (14), so that

$$
R=k \frac{\hat{\phi}}{\eta_{1}}, \text { where } \hat{\phi}=\beta_{1}+\beta_{2} \omega_{1}+\beta_{3} \omega_{3}+\beta_{4} \omega_{4}
$$

We choose $k$ as our bifurcation parameter, so that, if $k<k^{*}, R<1$ and if $k>k^{*}, R>1$. For $R=1$, we have $k=k^{*}=\eta_{1} / \hat{\phi}$.

We shall denote $J_{E_{0}}$ by $J_{k *}$ when $k=k^{*}$. The Jacobian matrix $J_{k *}$ has a right eigenvector $\mathbf{Z}=\left(z_{1}, z_{2}, z_{3}, z_{4}, z_{5}, z_{6}\right)^{\mathrm{T}}$ associated with the zero eigenvalue given by

$$
\begin{aligned}
& z_{1}=-\frac{k^{*}}{\mu}\left(\beta_{1}+\beta_{2} m_{1}+\beta_{3} m_{3}+\beta_{4} m_{4}\right) z_{2}, \\
& z_{2}=z_{2}>0 \text { is free, } \\
& z_{3}=m_{1} z_{2}, \text { where } m_{1}=\left(\frac{\sigma}{\eta_{2}}\right), \\
& z_{4}=m_{2} z_{2}, \text { where } m_{2}=\left(\frac{\sigma \psi}{\eta_{2} \eta_{3}}\right), \\
& z_{5}=m_{3} z_{2}, \text { where } m_{3}=\left(\frac{\gamma_{1}}{\eta_{4}}+\frac{\sigma \gamma_{2} \psi}{\eta_{2} \eta_{3} \eta_{4}}\right), \\
& z_{6}=m_{4} z_{2}, \text { where } m_{4}=\frac{1}{\eta_{5}}\left(\rho_{1}+m_{1} \rho_{2}+m_{2} \rho_{3}+m_{3} \rho_{4}\right) .
\end{aligned}
$$

The corresponding left eigenvector of $J_{k^{*}}$ is given by $\mathbf{V}=\left(v_{1}, v_{2}, v_{3}, v_{4}, v_{5}, v_{6}\right)^{\mathrm{T}}$, where $v_{1}=0, v_{2}>0$ (can take any value) and

$$
\begin{aligned}
& v_{6}=\zeta_{1} v_{2}, \text { where } \zeta_{1}=\left(\frac{k^{*} \beta_{4}}{\eta_{5}}\right), \\
& v_{5}=\zeta_{2} v_{2}, \text { where } \zeta_{2}=\left(\frac{\rho_{4} k^{*} \beta_{4}}{\eta_{4} \eta_{5}}\right)+\frac{k^{*} \beta_{3}}{\eta_{4}}, \\
& v_{4}=\zeta_{3} v_{2}, \text { where } \zeta_{3}=\left(\frac{1}{\eta_{3}}\right)\left[\gamma_{2} \zeta_{2}+\rho_{3} \zeta_{1}\right], \\
& v_{3}=\zeta_{4} v_{2} \text { where } \zeta_{4}=\frac{1}{\eta_{2}}\left(\psi \zeta_{3}+\rho_{3} \zeta_{1}+k^{*} \beta_{2}\right) .
\end{aligned}
$$

We note that $J_{k^{*}}$ has at least one eigenvalue with zero as the real part. Thus, we can use the centre manifold theory for our analysis. 
Using Theorem 4.1 in Ref. [4], we determine the signs of

$$
a=\sum_{h, i, j=1}^{6} v_{h} z_{i} z_{j} \frac{\partial^{2} f_{h}}{\partial x_{i} \partial x_{j}}\left(E_{0}\right), \quad b=\sum_{h, i=1}^{6} v_{k} z_{i} \frac{\partial^{2} f_{h}}{\partial x_{i} \partial k}\left(E_{0}\right) .
$$

For a complete synopsis of the applications of the centre manifold theory to epidemics such as TB and HIV/AIDS, the reader is referred to Refs. [4,23,29].

We now compute the non-zero expressions for the second partial derivatives of $\mathcal{F}$ at the disease free equilibrium point, so that

$$
\begin{aligned}
& \frac{\partial^{2} f_{2}}{\partial x_{2} \partial x_{2}}=-2 \frac{k^{*} \beta_{1} \mu}{\Pi}, \quad \frac{\partial^{2} f_{2}}{\partial x_{3} \partial x_{3}}=-2 \frac{k^{*} \beta_{2} \mu}{\Pi}, \quad \frac{\partial^{2} f_{2}}{\partial x_{5} \partial x_{5}}=-2 \frac{k^{*} \beta_{3} \mu}{\Pi}, \\
& \frac{\partial^{2} f_{2}}{\partial x_{2} \partial x_{5}}=-2 \frac{k^{*} \mu}{\Pi}\left(\beta_{1}+\beta_{3}\right), \quad \frac{\partial^{2} f_{2}}{\partial x_{2} \partial x_{4}}=-2 \frac{k^{*} \beta_{1} \mu}{\Pi}, \quad \frac{\partial^{2} f_{2}}{\partial x_{2} \partial x_{3}}=-2 \frac{k^{*} \mu}{\Pi}\left(\beta_{1}+\beta_{2}\right), \\
& \frac{\partial^{2} f_{2}}{\partial x_{3} \partial x_{4}}=-2 \frac{k^{*} \beta_{2} \mu}{\Pi}, \quad \frac{\partial^{2} f_{2}}{\partial x_{3} \partial x_{5}}=-2 \frac{k^{*} \mu}{\Pi}\left(\beta_{2}+\beta_{3}\right), \quad \frac{\partial^{2} f_{2}}{\partial x_{2} \partial x_{6}}=-\frac{k^{*} \mu}{\Pi}\left(\beta_{1}+\beta_{4}\right), \\
& \frac{\partial^{2} f_{2}}{\partial x_{3} \partial x_{6}}=-\frac{k^{*} \mu}{\Pi}\left(\beta_{2}+\beta_{4}\right), \quad \frac{\partial^{2} f_{2}}{\partial x_{5} \partial x_{6}}=-\frac{k^{*} \mu}{\Pi}\left(\beta_{3}+\beta_{4}\right), \quad \frac{\partial^{2} f_{2}}{\partial x_{4} \partial x_{6}}=-\frac{k^{*} \beta_{4} \mu}{\Pi}
\end{aligned}
$$

Since all the non-zero partial derivatives of $\mathcal{F}$ are negative and $z_{i}>0$ for $i=2, \ldots, 6$, it follows from the above expressions that $a<0$.

The following second partial derivatives are used to evaluate $b$,

$$
\frac{\partial^{2} f_{2}}{\partial x_{2} \partial k}=\beta_{1}, \quad \frac{\partial^{2} f_{2}}{\partial x_{3} \partial k}=\beta_{2}, \quad \frac{\partial^{2} f_{2}}{\partial x_{5} \partial k}=\beta_{3} \quad \text { and } \quad \frac{\partial^{2} f_{2}}{\partial x_{6} \partial k}=\beta_{4} .
$$

We thus have

$$
b=v_{2}\left(z_{2} \beta_{1}+z_{3} \beta_{2}+z_{5} \beta_{3}+z_{6} \beta_{4}\right)>0 .
$$

We thus have the following theorem.

THEOREM 3.5. The endemic equilibrium point $E_{1}$ is locally asymptotically stable for $R>1$, when $R$ is close to 1 .

\section{Various scenarios}

We now discuss the various scenarios that the model can exhibit by considering the model reproduction number. We deliberately avoid writing down model equations for the various scenarios because ultimately the stability of the models will depend on the reproduction numbers that can easily be derived from expression (7).

\subsection{No screening and treatment $\left(\beta_{3}=0\right)$}

If we do not have carriers and any intervention, then our model reduces to a $\operatorname{SICAA}_{t}$ (a basic HIV/AIDS) staged model. This can be done equivalently by setting $\rho_{3}=\rho_{4}=\psi=\gamma_{1}=\gamma_{2}=$ 
$\gamma_{3}=0$. Obviously, $R_{0 T}=\nu_{2}=0$, and the model reproduction number reduces to

$$
R_{0}=\frac{k \beta_{1}}{\mu+\sigma+\rho_{1}}+\nu_{11} \frac{k \beta_{2}}{\mu+\rho_{2}}+\nu_{21} \frac{k \beta_{4}}{\mu+\delta_{1}},
$$

where $\nu_{11}=\sigma /\left(\mu+\sigma+\rho_{1}\right)$ and $\nu_{21}=\rho_{1} /\left(\mu+\sigma+\rho_{1}\right)+\left(\sigma /\left(\mu+\sigma+\rho_{1}\right)\right)\left(\rho_{2} /\left(\mu+\rho_{2}\right)\right)$.

Note that, in the absence of screening and individuals under treatment, $R<R_{0}$. Interventions such as screening and treatment will thus reduce the reproduction potential of the disease.

\subsection{No treatment $\left(\beta_{3}=0\right)$}

An important scenario is the case where there is screening but no treatment. This is a common scenario in poor resource setting where screening for HIV is common but with no provision of the medication. We thus additionally set $\rho_{4}=\gamma_{1}=\gamma_{2}=\gamma_{3}=0$. The model reproduction number reduces to

$$
R_{C}=\frac{k \beta_{1}}{\mu+\sigma+\rho_{1}}+\nu_{11}\left(\frac{k \beta_{2}}{\mu+\rho_{2}+\psi}\right)+\nu_{22}\left(\frac{k \beta_{4}}{\mu+\delta_{1}}\right)
$$

where

$$
\nu_{22}=\frac{\rho_{1}}{\mu+\sigma+\rho_{1}}+\nu_{11}\left[\frac{\rho_{2}}{\mu+\rho_{2}+\psi}+\left(\frac{\rho_{3}}{\mu+\rho_{3}}\right)\left(\frac{\psi}{\mu+\rho_{2}+\psi}\right)\right] .
$$

We note that as $\psi \rightarrow 0, R_{C} \rightarrow R_{0}$.

\subsection{Treatment in the absence of screened carriers}

This is the case where only treatment for primary HIV in the acute phase and AIDS stage is given. Therefore, we set $\rho_{3}=\psi=\gamma_{2}=0$ giving us

$$
R_{\mathrm{Tsc}}=R_{0 I}+\nu_{1}\left(\frac{k \beta_{2}}{\mu+\rho_{2}}\right)+\nu_{23} R_{0 T}+\nu_{33} R_{0 A}
$$

where

$$
\nu_{23}=\left(\frac{\gamma_{1}}{\mu+\sigma+\rho_{1}+\gamma_{1}}\right) \text { and } \nu_{33}=\frac{\rho_{1}}{\mu+\sigma+\rho_{1}+\gamma_{1}}+\nu_{1}\left(\frac{\rho_{2}}{\mu+\rho_{2}}\right)+\nu_{23}\left(\frac{\rho_{4}}{\mu+\rho_{4}}\right) .
$$

Also as $\gamma_{1}, \gamma_{3} \rightarrow 0, R_{T \mathrm{sc}^{\prime}} \rightarrow R_{0}$.

\subsection{Treatment in later stages: treating the AIDS cases only}

HAART is usually initiated in later stages of the disease when HIV positive people are at their most infectious stage. At present in Southern Africa, HAART is only given to AIDS individuals who have experienced AIDS-defining symptoms or those who have $\mathrm{CD} 4{ }^{+} \mathrm{T}$-cell count below 200 cells $/ \mathrm{mm}^{3}$, which is the recommended AIDS defining stage by World Health Organization. We therefore consider the effect of treating the AIDS cases only since it has already been pointed out that early treatment has not given any tangible advantage. This means that $\gamma_{1}=\gamma_{2}=0$. 
The reproduction number $R_{\mathrm{TA}}$, to denote late treatment, becomes

$$
\begin{aligned}
R_{\mathrm{TA}} & =\frac{k \beta_{1}}{\mu+\sigma+\rho_{1}}+\nu_{11} R_{0 C}+\nu_{24} R_{0 A} \\
\nu_{24} & =\frac{\rho_{1}}{\mu+\sigma+\rho_{1}}+\nu_{1}\left[\frac{\rho_{2}}{\mu+\rho_{2}+\psi}+\left(\frac{\rho_{3}}{\mu+\rho_{3}}\right)\left(\frac{\psi}{\mu+\rho_{2}+\psi}\right)\right] .
\end{aligned}
$$

We note that there will not be any contribution from the treated class since treated individuals from the AIDS class do not join the treated class but rather the treated AIDS class which does not contribute to the spread of the epidemic.

\subsection{Treatment in early stages: treating the normal infectives and screened carriers}

This is our main model which includes all the above cases and in addition, early treatment to anyone in the normal infectives who can afford it. It is well known that patients seek treatment from private doctors who can start the HIV infected individuals even if their CD4 ${ }^{+} \mathrm{T}$-cell count is above 200 cells $/ \mathrm{mm}^{3}$. The reproduction number is given by expression (7). It can be shown that this is the smallest reproduction number, so that $R<R_{\mathrm{Tsc}^{\prime}}<R_{\mathrm{TA}}<R_{0}<R_{C}$. We now carry out numerical simulations to assess the impact of the different parameters on the epidemic.

\section{Numerical simulations}

Mathematical models often include parameters, estimated from experiments, for which their actual values are not known precisely [19]. Therefore, in this section, we consider the model predictions of the basic reproduction number of the infection for some specific parameter values chosen from the Central Statistical Office of Zimbabwe (CSZ) and in Ref. [18]. The numerical values are tabulated in Table 1.

We begin by considering the reproduction numbers and how they are influenced by the model parameters. The full qualitative analysis of the reproduction number of our model is hindered by the complexity of the expressions for the various reproduction numbers. We have however tried to explain in detail the meanings of each of the terms that constitute the model reproduction number. We therefore use numerical simulations to illustrate the relationship and behaviour of the various reproduction numbers to compliment our findings. We consider approximate values of important parameters in the prediction of the reproduction numbers of infection and vary the number of sexual partners $k$, since this affects transmission rates and the screening rate $\psi$, of carriers.

Figure 1 illustrates the behaviour and relationship of the different reproduction numbers as the number of partners $k$, increase using parameter values in the Table 1 . The trends confirm the

Table 1. Table showing the numerical values of parameters from the CSZ and literature.

\begin{tabular}{llll}
\hline Parameter & Approximate value/year & Parameter & Approximate value/year \\
\hline$\Pi$ & $\Pi \geq 10,000$ people & $\sigma$ & $0.05 \leq \sigma \leq 0.3$ \\
$\mu$ & $0.01 \leq \mu \leq 0.025(\mathrm{CSZ})$ & $c$ & $1 \leq c \leq 10$ \\
$\beta_{1}$ & $0 \leq \beta_{1} \leq 1$ & $\beta_{2}$ & $0 \leq \beta_{2} \leq 1$ \\
$\beta_{3}$ & $0 \leq \beta_{3} \leq 1$ & $\beta_{4}$ & $0 \leq \beta_{4} \leq 1$ \\
$\gamma_{1}$ & $0.06 \leq \gamma_{1} \leq 0.2$ & $\psi$ & $0.3 \leq \psi \leq 0.6$ \\
$\gamma_{2}$ & $0.1 \leq \gamma_{2} \leq 0.3$ & $\gamma_{3}$ & $0.1 \leq \gamma_{3} \leq 0.4$ \\
$\delta_{1}$ & $0.3 \leq \delta \leq 0.36$ & $\rho_{1}$ & $0.03 \leq \rho_{1} \leq 0.1$ \\
$\rho_{2}$ & $0.06 \leq \rho_{2} \leq 0.45$ & $\rho_{3}$ & $0.2 \leq \rho_{3} \leq 0.4$ \\
\hline
\end{tabular}




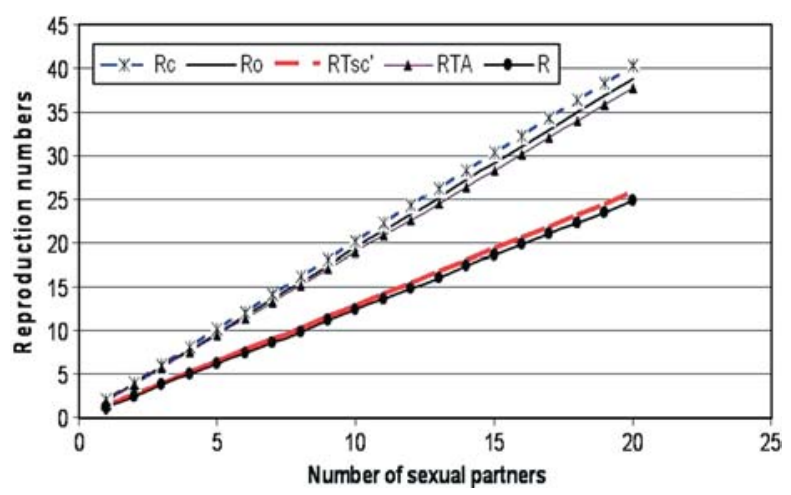

Figure 1. The diagram shows how various reproduction numbers evolve with increasing numbers of sexual partners. It is important to note the for the reproduction numbers in the legend, $R T=R_{\mathrm{T}}$, $R T s c^{\prime}=R_{\mathrm{Tsc}^{\prime}}$ and $R T A=R_{\mathrm{TA}}$.

analytic result $R<R_{\mathrm{Tsc}^{\prime}}<R_{\mathrm{TA}}<R_{0}<R_{C}$, for the given set of parameter values. We note that the presence of carriers without any treatment increases the basic reproduction number.

Figure 2 shows the effect of increased screening on the reproduction numbers $R_{\mathrm{TA}}$ and $R$. The graph shows the importance of encouraging screening in the fight against HIV/AIDS. We can also use such an approach to determine the threshold screening rate $\psi^{*}$, (the value of $\psi$ above which we can effectively bring the model reproduction number below unit). We note in Figure 2 that the optimal screening rate that would bring the model reproduction number to a value below one, should give an $80 \%$ coverage for the given parameter values.

To further investigate the role of screening, we consider a hypothetical population of initially 500,700 individuals, initial conditions $\left(S(0), I(0), C(0), C_{\mathrm{s}}(0), T(0), A(0)\right)=(465,000,20,000$, $10,000,5000,500,200)$. We consider a scenario where interventions to an on going epidemic are carried out. We present a situation where screening in introduced as a control measure in the fight against HIV/AIDS after a period of 50 years from the start of the epidemic. The changes of the populations of the carriers and screened carriers are tracked for $\psi / 2, \psi, 3 \psi / 2,2 \psi$ and $3 \psi$ starting with $\psi=0.2$. The last two cases, $2 \psi$ and $3 \psi$ are equivalent to doubling and trebling the screening rate. If in this particular setting, $\psi$ is doubled, the number of screened carriers increases by about $13.6 \%$. The results are presented in Figure 3.

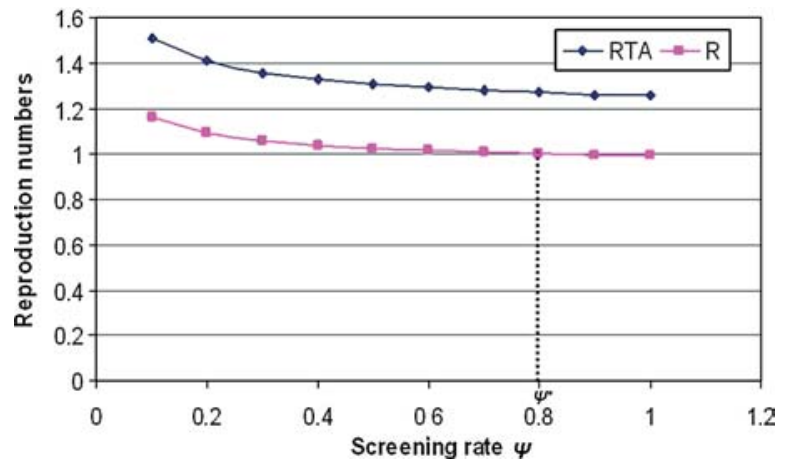

Figure 2. The diagram shows the evolution of the reproduction numbers $R_{\mathrm{TA}}$ and $R$ as the screening rate $\psi$ changes for the following parameter values: $k=1, \beta_{1}=0.2, \beta_{2}=0.08, \beta_{3}=0.02, \beta_{4}=0.19, \gamma_{1}=0.08$, $\gamma_{2}=0.15, \quad \gamma_{3}=0.2, \quad \rho_{1}=0.001, \rho_{2}=0.1, \quad \rho_{3}=0.2, \quad \rho_{4}=0.5, \quad \sigma=0.2, \mu=0.02$ and $\delta_{1}=0.33$. The reproduction number in the legend, $R T A=R_{\mathrm{TA}}$. 

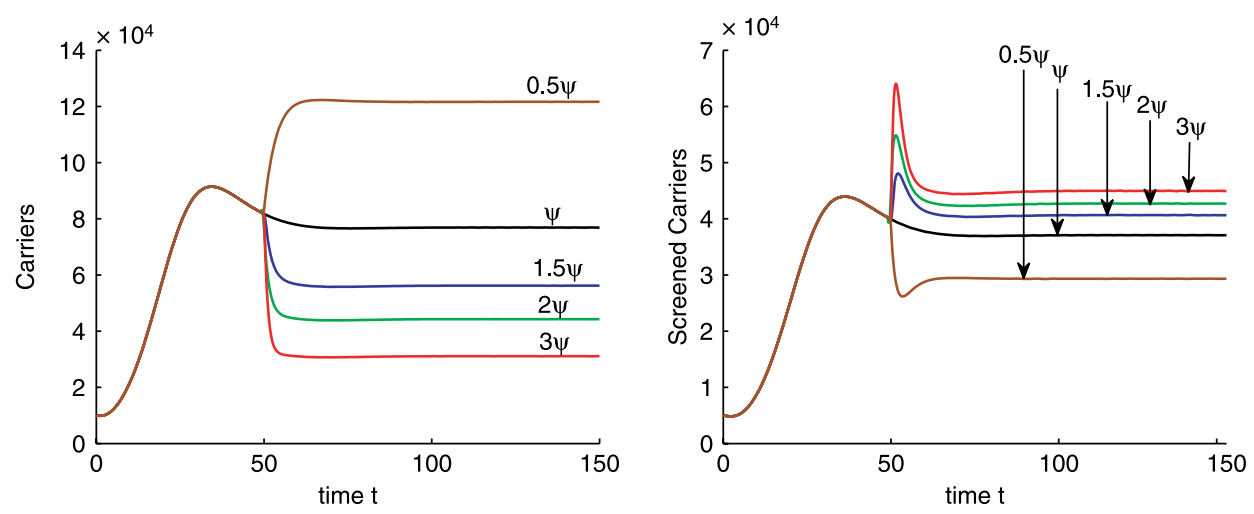

Figure 3. The diagrams track the changes in the populations of the carriers when intervention is instituted after 50 years from the onset of the epidemic for the following parameter values: $k=1.5, \beta_{1}=0.3$, $\beta_{2}=0.1, \beta_{3}=0.09, \beta_{4}=0.2, \mu=0.022, \pi=100,000, \sigma=0.2, \rho_{1}=0.001, \rho_{2}=0.09, \rho_{3}=0.45$, $\rho_{4}=0.2, \gamma_{1}=0.3, \gamma_{2}=0.15, \gamma_{3}=0.3$ and $\delta=0.33$.

Figure 4 shows that the system settles at an endemic steady state with a reproduction number $R=1.7895$ for the given parameter values in the caption. This confirms the local stability of the endemic equilibrium point whenever the reproduction number $R$ is above unit. The prevalence curve for the same parameter values is given in Figure 5.

For the given parameter values, the prevalence of the disease, that is, the proportion of individuals in a population who have the disease at a specific instant, will be around $22 \%$. This describes scenario in Southern African countries [28]. If individuals with AIDS are not treated (setting $\gamma_{3}=0$ ), the reproduction number increases, so that $R=2.1282$ and the endemic equilibrium population of these individuals also increases. This however is not inline with the current observed trends. The HIV prevalence in Southern Africa is now showing a downward
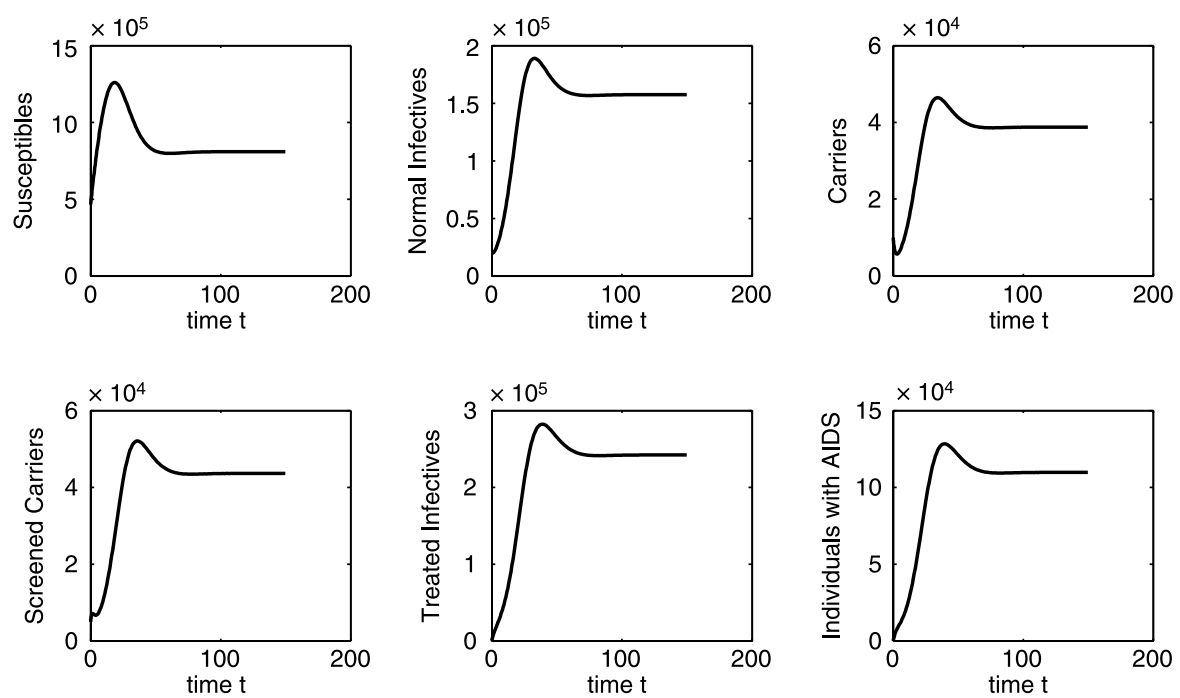

Figure 4. The diagrams shows that the various populations settle at an endemic steady state for the following parameter values: $k=1.5, \beta_{1}=0.3, \beta_{2}=0.09, \beta_{3}=0.08, \beta_{4}=0.2, \mu=0.022, \pi=100,000$, $\sigma=0.2, \rho_{1}=0.001, \rho_{2}=0.09, \rho_{3}=0.45, \rho_{4}=0.2, \psi=0.2, \gamma_{1}=0.3, \gamma_{2}=0.15, \gamma_{3}=0.3$ and $\delta=0.33$. 


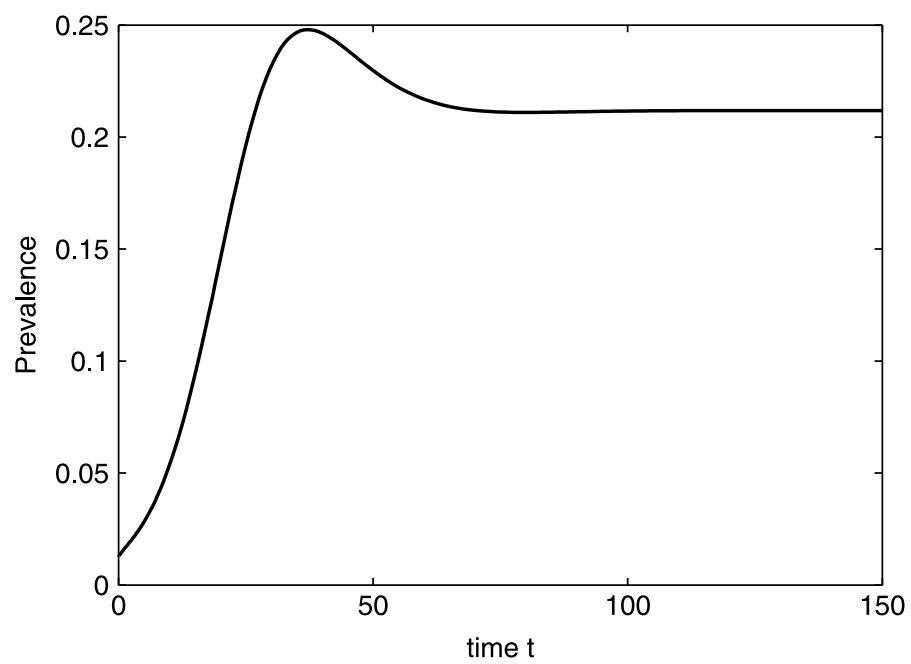

Figure 5. The prevalence curve of the model for the parameter values given for Figure 4.

trend especially in Zimbabwe with stabilization observed in the other countries [27]. By prevalence, we mean the total number of HIV cases in the population at a given time expressed as a ratio of the infected individuals to the total population. We now consider the variation of the number of infected individuals as the average number of sexual partners change per given time. The corresponding values of the reproduction number are as shown in Figure 6.

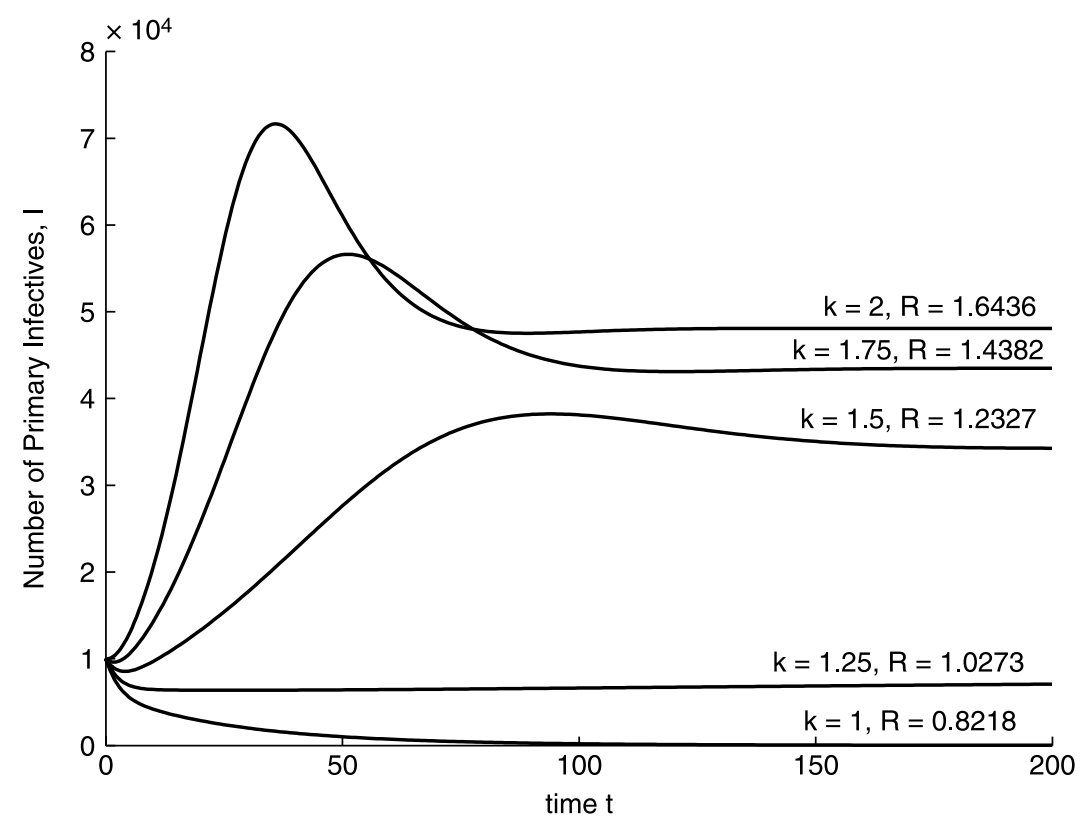

Figure 6. The figure shows how the number of infectives and the reproduction number $R$, change with increasing numbers of sexual partners for the following parameter values: $\beta_{1}=0.2, \beta_{2}=0.07, \beta_{3}=0.06$, $\beta_{4}=0.2, \mu=0.02, \pi=20,000, \sigma=0.1,0.2,0.45, \rho_{1}=0.001, \rho_{2}=0.45, \rho_{3}=0.4, \rho_{4}=0.5, \psi=0.4$, $\gamma_{1}=0.08, \gamma_{2}=0.15, \gamma_{3}=0.1$ and $\delta=0.33$. 


\section{Discussion and conclusion}

The results of this study provide some insights on the benefits of including the carriers in HIV/AIDS model development and their potential impact on disease transmission dynamics. The qualitative features of the model were investigated. The model reproduction number $R$ was determined and its discussion given to highlight the contribution of each infectious class to the disease dynamics. The model has two equilibria, the disease free equilibrium point, $E_{0}$ and the endemic equilibrium point, $E_{1}$. The results showed that the disease free equilibrium point is stable (locally and globally) for $R<1$ and endemic equilibrium point is locally stable for $R>1$. The local stability of the endemic equilibrium point was proved using the centre manifold theory. By setting some parameters to zero, the model exhibited a number of scenarios that gave rise to six reproduction numbers. An analytical comparison of the reproduction numbers was also done.

The potential danger posed by carriers has been shown. The objective of disease control and prevention is to lower the basic reproduction number $R_{0}$ to a level less than unit, so that the disease clears. Our objective was also to determine the role of screening disease carriers and the potential impact of increased screening coverage. The effect of the screening rate $\psi$ on the reproduction numbers of the model and the case where treatment is offered to individuals with AIDS only was investigated. We showed that the presence of randomly screened carriers further reduces the endemicity of the disease, since the basic reproduction number $R_{0}$ is further reduced. Further treatment in the presence of screening reduces the spread of the disease as compared to treatment without screening. Screening helps to counsel and educate carriers on safe sexual interaction methods and encourage them to seek treatment since there is a lot of fear of the side effects of using ARV therapy or HAART among people living with HIV/AIDS. Screening is also important, especially in Southern Africa where many individuals cannot afford HAART medication. They are likely to benefit from counselling, as they will learn about various ways of preventing further infection. Similar deductions were made in Ref. [26].

Our study also shows that the optimal screening rate that would bring the model reproduction number to a value below one, should give an $80 \%$ coverage for the given parameter values. In tracking the changes of the number of individuals in the screened class, we noted that doubling the screening rate leads to a $13.6 \%$ in the number of screened cases. Other interventions can be investigated in a similar fashion. The results clearly show that screening alone cannot be used as a comprehensive HIV fighting strategy. Multiple strategies should therefore be emphasized in the fight against HIV/AIDS (see Ref. [18]). The equilibrium levels on normal infectives and AIDS can be maintained at desired levels. A sizeable number of people living with HIV/AIDS cannot afford treatment because of cost of ARV drugs and the drugs supply has rarely kept pace with demand. Introducing early treatment can also be beneficial as we note that this gives the smallest reproduction number.

Numerical simulations using data from the CSZ were carried out. A comparison of the reproduction numbers arising from the various scenarios was done numerically and similar results were obtained. Our results also highlight the dangers of increasing the number of sexual partners in the spread of HIV and the corresponding changes to the model reproduction number.

Finally, this study shows that using the interventions analysed in this study makes the prospects of controlling HIV bright. While the use of HAART appears to be more effective in terms of preventing new cases in the long run, it should be emphasized that ARVs are not readily available, especially in developing countries. Affordable interventions, such as educational programs like random screening and counselling can lower the incidence and prevalence levels. 
This helps people to have a positive attitude towards preventive methods against infection. In conclusion, multiple strategies implemented in combination can be very effective in controlling the spread of the disease with screening and testing, playing an important role both as a preventive measure and a barometer for success in the fight against HIV.

\section{Acknowledgements}

The first author acknowledges, with thanks, the support of Africa Millennium Mathematics and Science Initiative (AMMSI) and National University of Science and Technology (NUST) for sponsoring and supporting her research visit. The second author acknowledges, with gratitude, the support by South African Center of Epidemiological Modeling and Analysis (SACEMA) in the production of this manuscript. The authors are also grateful to the anonymous reviewers for their patience and very useful comments that improved the paper significantly.

\section{References}

[1] N.T. Bailey, The Mathematical Theory of Infectious Diseases, 2nd ed., Charles Griffin, London, 1975.

[2] S.M. Blower, G.J.P. van Griensven, and E.H. Kaplan, An analysis of the process of human immunodeficiency virus sexual risk behaviour change, Epidemiology 6 (1995), pp. 238-242.

[3] S.P. Blythe and R.M. Anderson, Variable infectiousness in HIV transmission models, Math. Med. Biol. 5 (1988), pp. 181-200.

[4] C. Castillo-Chavez and B. Song, Dynamical models of tuberculosis and their applications, Math. Biosci. Eng. 1 (2004), pp. 361-404.

[5] L. Esteva-Peralta and J.X. Velasco-Hernandez, M-Matrices and local stability in epidemic models, Math. Comput. Model. 36 (2002), pp. 491-501.

[6] G.P. Garnet, An introduction to mathematical models in sexually transmitted disease epidemiology, Sex. Transm. Infect. 78 (2002), pp. 7-12.

[7] D.D. Ho, Time to HIV, early and hard, N. Engl. J. Med. 333 (1995), pp. 450-451.

[8] T. Horn, Treating HIV infection - Is there a benefit?, Available at http://www.aidsmeds.com.

[9] E. Kupek, Estimation of the number of sexual partners for the nonrespondents to a large national survey, Arch. Sex. Behav. 28 (1999), pp. 233-242.

[10] S.E. Langford, J. Ananworanich, and D.A. Cooper, Predictors of disease progression in HIV infection: a review, AIDS Res. Ther. 4 (2007), pp. 1-14.

[11] I.G. Lauko, Stability of disease free states in epidemic models, Math. Comput. Model. 43 (2006), pp. $1357-1366$.

[12] L.S. Luboobi, Mathematical models for the dynamics of AIDS epidemic, Biometry for Development, 1991, pp. 76-83.

[13] E.M. Lungu, M. Kgosimore, and F. Nyabadza, Models for the spread of HIV/AIDS: Trends in Southern Africa, in Mathematical studies on Human Disease Dynamics: Emerging Paradigms and Challenges, A.B. Gumel, C. Castillo-Chavez, R.E. Mickens, and D.P. Clemence, eds., AMS, Boston, 2006, pp. 259-277.

[14] M.S. Moghadas, A.B. Gumel, R.G. McLeod, and R. Gordon, Could condoms stop the AIDS epidemic?, J. Theor. Med. 5 (2003), pp. 171-181.

[15] S.D. Musekwa, Development of deterministic and stochastic epidemic models with special reference to HIV/AIDS, DPhil. diss., University of Zimbabwe, 2005.

[16] J.Y.T. Mugisha, F. Baryarama, and L.S. Luboobi, Modelling HIV/AIDS with complacency in a population with declining prevalence, Comput. Math. Methods Med. 7 (2006), pp. 27-35.

[17] D.J. Nokes and R.M. Anderson, The use of mathematical models in the epidemiological study of infectious diseases and in the design of mass immunization programmes, Epidemiol. Infect. 101 (1988), pp. 1-20.

[18] F. Nyabadza, A mathematical model for combating HIV/AIDS in Southern Africa, J. Biol. Syst. 14 (2006), pp. 357-372.

[19] A. Oxenius, D.A. Price, P.J. Easterbrook, C.A. O'Callaghan, A.D. Kelleher, J.A. Whelan, G. Sontag, A.K. Sewell, and R.E. Phillips, Early highly active antiretroviral therapy for acute HIV-1 infection preserves immune function of $C D 8^{+}$and $C D 4^{+}$T lymphocytes, PNAS 97 (2000), pp. 3382-3387.

[20] S. Portsmouth, N. Imami, A. Pires, J. Stebbing, J. Hand, M. Nelson, F. Gotch, and B.G. Gazzard, Treatment of primary HIV-1 infection with nonnucleoside reverse transcriptase inhibitor-based therapy is effective and well tolerated, HIV Med. 5 (2004), pp. 26-29. 
[21] M.S. Sanchez, R.M. Grant, T.C. Porco, K.L. Gross, and W.M. Getz, A decrease in drug resistance levels of the HIV epidemic can be bad news, Bull. Math. Biol. 67 (2005), pp. 761-782.

[22] S.H. Schmitz, Effects of treatment or/and vaccination on HIV transmission in homosexual with genetic heterogeneity, Math. Biosci. 167 (2000), pp. 1-18.

[23] O. Sharomi, C.N. Podder, A.B. Gume, and B. Song, Mathemtical analysis of the transmission dynamics of HIV/TB co-infection in the presence of treatment, Math. Biol. Eng. 5 (2008), pp. $145-174$.

[24] D.E. Smith, B.D. Walker, D.A. Cooper, E.S. Rosernberg, and J.M. Kaldor, Is antiretroviral treatment of primary HIV infection clinically justified on the basis of current evidence?, AIDS 18 (2004), pp. 709-718.

[25] H.R. Thieme, Persistence under relaxed point-dissipativity (with applications to an endemic model), SIAM J. Math. Anal. 24 (1993), pp. 407-435.

[26] A. Tripathi, R. Naresh, and D. Sharma, Modelling the effect of screening of unaware infectives on the spread of HIV infection, Appl. Math. Comput. 184 (2007), pp. 1053-1068.

[27] UNAIDS-WHO, AIDS epidemic update (2007), pp. 4-17.

[28] UNAIDS-WHO, Fact sheet (2006) (20061121_epi_fs_ssa_en.pdf). Available at http://data.unaids. org/pub/EpiReport/2006.

[29] P. van den Driessche and J. Watmough, Reproduction numbers and sub-threshold endemic equilibrium for compartmental models of disease transmission, Math. Biosci. 180 (2002), pp. 29-48. 


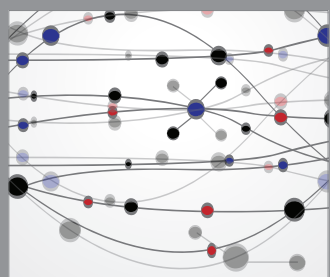

The Scientific World Journal
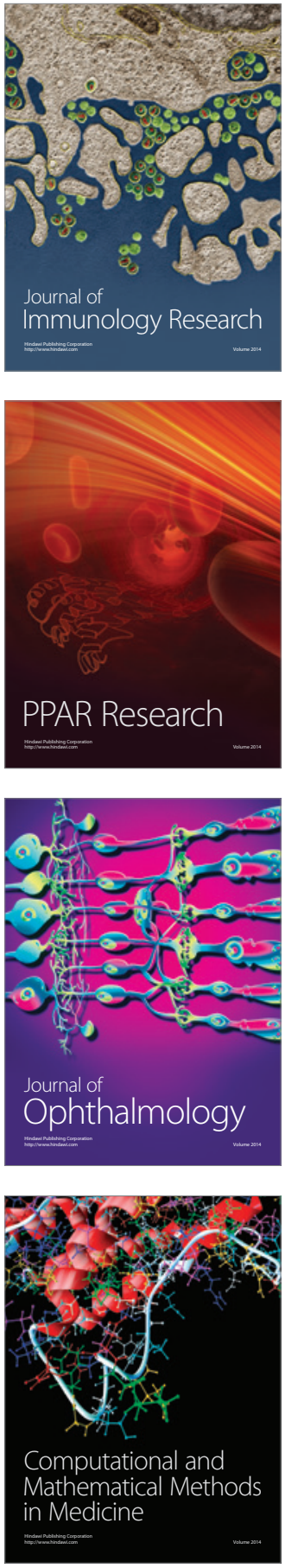

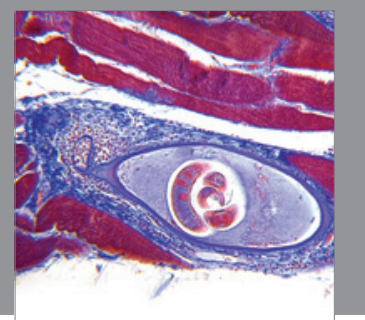

Gastroenterology

Research and Practice
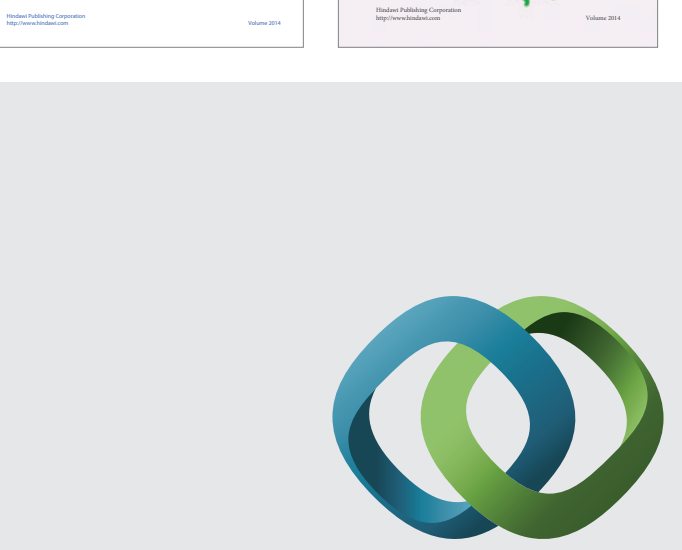

\section{Hindawi}

Submit your manuscripts at

http://www.hindawi.com
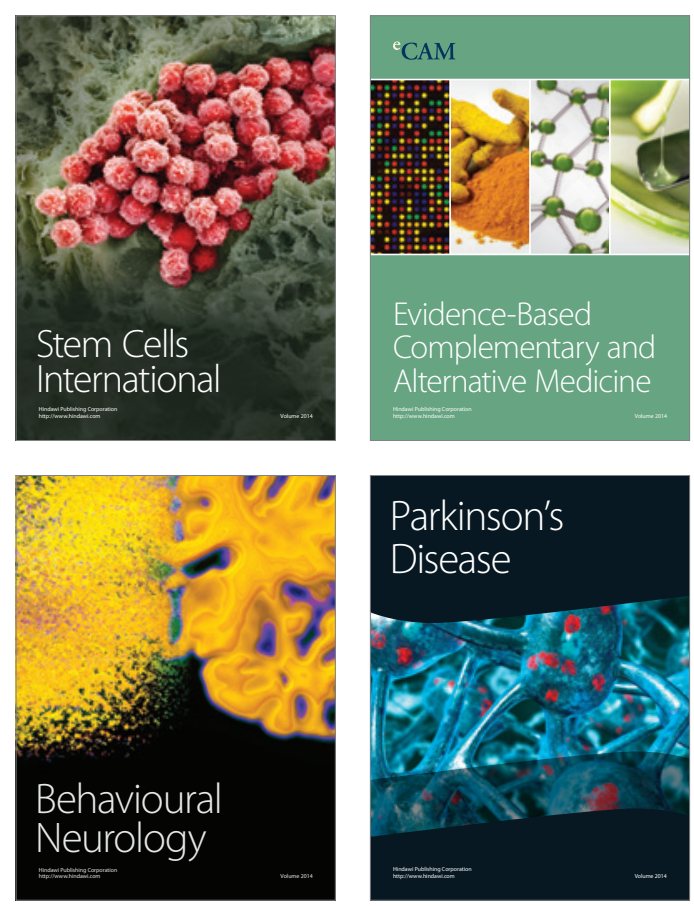

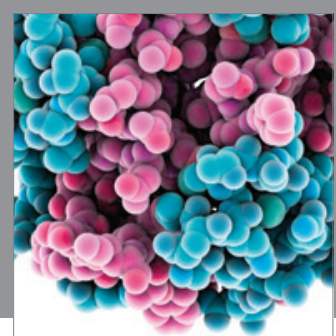

Journal of
Diabetes Research

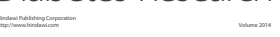

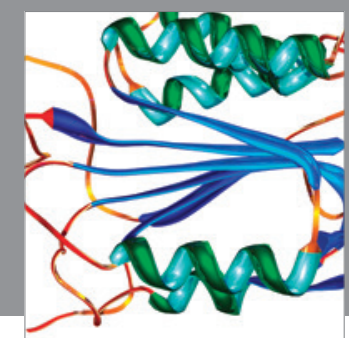

Disease Markers
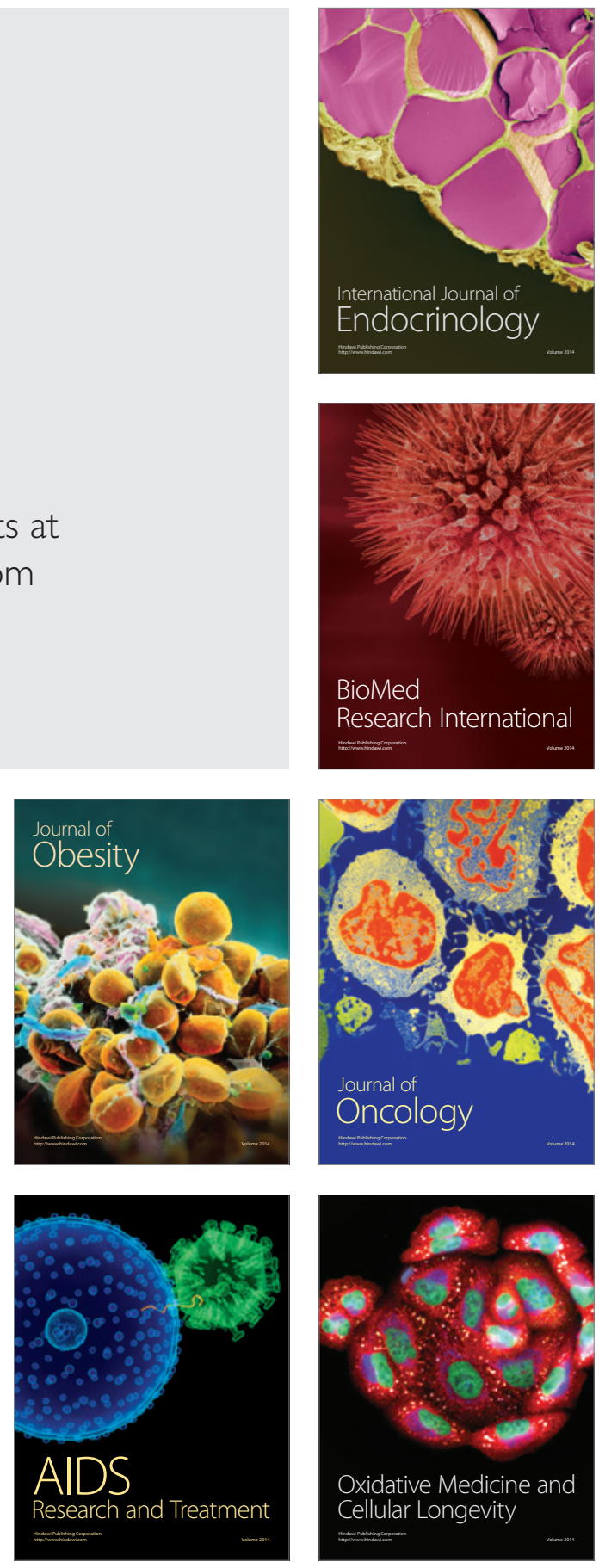